\title{
Sudden cardiac death in young people and athletes
}

\author{
Domenico Corrado, Alessandro Zorzi \\ Department of Cardiac, Thoracic and Vascular Sciences, University of Padua, Italy
}

\begin{abstract}
The sudden death of a young subject or athlete is a rare but tragic event. The most common mechanism of cardiac arrest leading to sudden death is abrupt ventricular fibrillation as a consequence of an underlying cardiovascular disease. The culprit diseases are often clinically silent and unlikely to be suspected or diagnosed on the basis of spontaneous symptoms. The longrunning Italian experience with medical evaluation of young individuals before their participation in sports has provided compelling evidence that screening the young populations for at-risk cardiac diseases offers the potential to identify asymptomatic athletes who have potentially lethal cardiovascular abnormalities and may protect them from the risk of sudden death. In this review we will discuss cardiovascular causes of sudden death in young people and athletes with particular emphasis on pathological findings and pathophysiology of sudden cardiac arrest. Occurrence of prodromal symptoms, possible early diagnosis at preparticipation screening and early defibrillation in the sports arenas will also be addressed.
\end{abstract}

\section{Introduction}

The sudden death (SD) of a young subject or athlete, though rare, is a tragic event, which devastates families, institutions (high school, college, or professional organization), the community, and sports medicine team. It is widely publicized by media with the implication that such a fatality is preventable. The beginning of the year 2018 was notorious for SD in young athletes, with three tragic events occurring in top-level athletes including a 31-year-old Italian soccer player, a 25-yearold Croatian soccer player, and a 22-year-old Belgian cyclist. These events have revived the debate regarding

Correspondence: Domenico Corrado, Department of Cardiac, Thoracic and Vascular Sciences, University of Padova Medical School, via Giustiniani 2, 35121 Padova, Italy.

Tel.: +39.049.8212458 - Fax: +39.049.8212309.

E-mail: domenico.corrado@unipd.it

Key words: Sudden death; ventricular fibrillation; cardiomyopathy; sports cardiology; electrocardiogram.

Contributions: the authors contributed equally.

Conflict of interest: the authors declare no potential conflicts of interest.

Received for publication: 12 April 2018.

Accepted for publication: 18 April 2018.

This work is licensed under a Creative Commons Attribution NonCommercial 4.0 License (CC BY-NC 4.0).

(C) Copyright D. Corrado and A. Zorzi, 2018

Licensee PAGEPress, Italy

Italian Journal of Medicine 2018; 12:74-87

doi:10.4081/itjm.2018.1027 the need for a cardiovascular evaluation of young individuals embarking on the career of athletes and the best screening protocol.

The most common mechanism of cardiac arrest leading to SD is abrupt ventricular fibrillation as a consequence of an underlying cardiovascular disease. ${ }^{1}$ The culprit diseases are often clinically silent and unlikely to be suspected or diagnosed on the basis of spontaneous symptoms. ${ }^{2}$ The long-running Italian experience with medical evaluation of young individuals before their participation in sports has provided compelling evidence that screening the young populations for at-risk cardiac diseases offers the potential to identify asymptomatic athletes who have potentially lethal cardiovascular abnormalities and may protect them from the risk of SD. ${ }^{3}$

This article reviews the epidemiology, the causes and the mechanisms of SD in young people and athletes and addresses risk stratification and prevention strategies.

\section{Epidemiology}

\section{Incidence}

The assessment of the precise frequency of SD is hampered by the retrospective nature of most analyses. The risk of SD generally increases with age and is greater in men. In the general population of middle-age and elderly subjects the estimated rate of $\mathrm{SD}$ ranges from 1-2/1000/year; in comparison, a significantly lower incidence of fatal events has been reported in young people and athletes ( $\leq 35$ years). ${ }^{3-29}$ A prospective study in Italy reported a cumulative annual incidence of SD of about 1 per 100,000 young individuals. ${ }^{3}$ Among the non-athletic young people the incidence was $0.9 / 100,000 /$ year, whereas the prevalence of fatalities among young competitive 
athletes was estimated to be approximately 2.3 per 100,000 athletes per year. Thus, the incidence of sudden death in athletes was 2.5 -fold greater, indicating that physical activity increases the risk of sudden death in this age group.

\section{Etiology}

Although the final pathway of sudden death is cardiac arrest, the involved mechanisms may be of cerebral, respiratory or cardiac origin. ${ }^{30}$ Cerebral sudden death in young people is usually caused by a cerebral hemorrhage, either intraparenchymal or subarachnoid, due to a rupture of a congenital berry aneurysm. Respiratory sudden death is the consequence of a sudden and severe obstruction of airways, most often caused by an abrupt bronchospasm in a young subject with allergic asthma.

Cardiovascular sudden death is unfrequently caused by mechanical reasons (mechanical $S D$ ) such as a spontaneous laceration or dissection of the ascending aorta with rupture into pericardial cavity and cardiac tamponade; the basic heart defect is an elastic fragmentation of the aortic tunica media with cystic medial necrosis, that most frequently occurs in association with bicuspid aortic valve or isthmic coarctation, or in the setting of Marfan syndrome.

Instead, the vast majority of young SD victims have an underlying structural heart disease, which provides a substrate for ventricular tachycardia/ fibrillation leading to cardiac arrest (electrical SD).

Atherosclerotic coronary artery disease is the most common cause of SD in adults and elderly subjects. Epidemiologic studies support the concept that in this age group habitual physical activity may offer protection over the long-term against cardiovascular events. In fact, regular exercise prevents development and progression of atherosclerotic coronary artery disease by favorable effects on lipid metabolism and weight reduction, and may enhance both coronary artery plaque and myocardial electrical stability. ${ }^{31-33}$

Unlike older subjects, a broad spectrum of cardiovascular substrates (including congenital and inherited heart disorders) may underlie SD in young people and athletes (age $\leq 35$ years) (Table 1). ${ }^{1}$ Adolescent and young adults involved in physical activity have approximately a 3 times greater risk of SD than their non-athletic counterparts (Figure 1). ${ }^{5}$ However, sport is not itself the cause of the enhanced mortality; rather, it acts as a trigger of cardiac arrest in those athletes who are affected by cardiovascular conditions which predispose to life-threatening ventricular arrhythmias during physical exercise. This reinforces the need for systematic cardiovascular evaluation of adolescent and young individuals, especially those embarking on physical activity, with the aim to identify those with potentially lethal cardiovascular diseases and protect them against the increased risk of sudden death.

\section{Cardiomyopathies}

Cardiomyopathies have been consistently implicated as a leading cause of sports-related cardiac arrest in young people and athletes. Hypertrophic cardiomyopathy (HCM) has been reported to account for more than one third of fatal cases in the United States and arrhythmogenic right ventricular cardiomyopathy/dysplasia (ARVC) for approximately one fourth in the Veneto Region of Italy. ${ }^{5}$ Other cardiovascular substrates for sports-related SD in the young include premature atherosclerotic coronary artery disease, congenital coronary anomalies, myocarditis, dilated cardiomyopathy, mitral valve prolapse (MVP), conduction system diseases and WPW syndrome. ${ }^{30}$

Hypertrophic cardiomyopathy, the most common inherited heart muscle disease, is caused by mutations of genes encoding sarcomeric proteins. The phenotype of HCM is characterized by left ventricular hypertrophy, which occurs in the absence of abnormal

Table 1. Cardiovascular causes of sudden death in different age-groups.

Age $\geq 35$ years

Atherosclerotic coronary artery disease

Age $<35$ years

Hypertrophic cardiomyopathy

Arrhythmogenic right ventricular cardiomyopathy/dysplasia

Premature coronary atherosclerosis

Congenital anomalies of coronary arteries

Myocarditis

Aortic rupture

Valvular disease

Preexcitation syndromes and conduction diseases

Ion channel diseases

Congenital heart disease, operated or unoperated

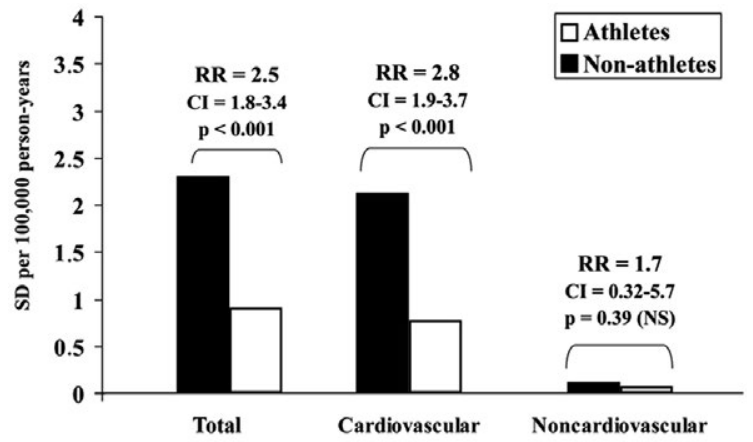

Figure 1. Incidence and relative risk (RR) of sudden death (SD) among young athletes and non-athletes from total, cardiovascular and non-cardiovascular causes. See the text for details. CI, confidence interval; NS, not significant. Adapted from Corrado et al., $2003^{5}$ with permission. 
loading conditions. Left ventricular (LV) hypertrophy may be either symmetric or asymmetric and is associated with the reduction in LV chamber size with increased myocardial stiffness, which may critically impair diastolic LV filling and intramural coronary blood flow. Dynamic LV outflow tract obstruction is also demonstrable at rest or during exercise in a large proportion of patients. The histopathologic hallmark of HCM is myocardial disarray with widespread, bizarre and disordered arrangement of myocytes usually associated with interstitial and/or replacementtype fibrosis (Figure 2A). ${ }^{34}$ Myocardial fibrosis is an acquired phenomenon, in part related to abnormalities of the intramural coronary arteries with luminal narrowing (small vessel disease), which intervenes in the natural history of the disease and contributes to the arrhythmogenicity. HCM has been implicated as the principal cause of SD during sports in the United States. Cardiac arrest has been attributed to ventricular fibrillation, which is triggered by the interaction between adrenergic stimulation, such as that occurring during physical exercise, and the underlying, electrically instable cardiomyopathic substrate. The diagnosis traditionally relies on the demonstration by echocardiography of otherwise unexplained diffuse or segmental LV wall and/or septal thickening $\geq 15 \mathrm{~mm}$; a lower cut-off value $(\geq 13 \mathrm{~mm})$ is used in the context of family members of affected individuals, especially if they are mutation carriers. Cardiac magnetic resonance imaging may provide additional diagnostic and prognostic information given its ability to better detect segmental LV hypertrophy in some regions, such as anterior free wall, posterior septum or apex, which are not visualized adequately by echocardiography. Moreover, it allows a more accurate wall thickness measurements and identification of areas of intramyocardial late gadolinium enhancement corresponding to scar tissue. Affected patients are at increased risk of ventricular arrhythmias and SD, heart failure, and thromboembolism. ${ }^{35-37}$ Electrocardiogram (ECG) abnormalities such as an increased QRS voltage, pathologic "Q" waves, and repolarization changes have been reported in up to $95 \%$ of patients with HCM. ${ }^{38}$ This explains why preparticipation ECG screening of young competitive athletes, which has been in practice in Italy since 1982, has allowed successful identification and mortality reduction of athletes with $\mathrm{HCM}^{39}$

Arrhythmogenic right ventricular cardiomyopathy is an inherited cardiomyopathy characterized by progressive loss of myocytes and replacement by fibrofatty tissue, which creates the substrate for life-threatening ventricular arrhythmias (Figure 2B). In its classical form the phenotypic manifestations predominantly involve the right ventricle (RV), but biventricular and left-dominant variants exist. Molecular genetic studies showed that ARVC is a genetic disorder resulting from defective desmosomal proteins, most often with an autosomal dominant pattern of inheritance. ${ }^{40,41}$ The clinical manifestations of the disease usually occur between the second and forth decade of life and consist of ECG depolarization and repolarization changes, typically localized in the right precordial leads (V1-V3/V4), structural abnormalities, such as global or regional dilatation/dysfunction of the $\mathrm{RV}$ and, most importantly, ventricular arrhythmias with a left bundle branch block morphology, ranging from isolated premature ventricular beats to sustained ventricular tachycardia, which can degenerate into ventricular fibrillation. ${ }^{42,43}$ The diagnosis of ARVC requires a combination of different criteria from various categories such as: i) histopathological abnormalities (fibro-fatty myocardial replacement) at endomyocardial

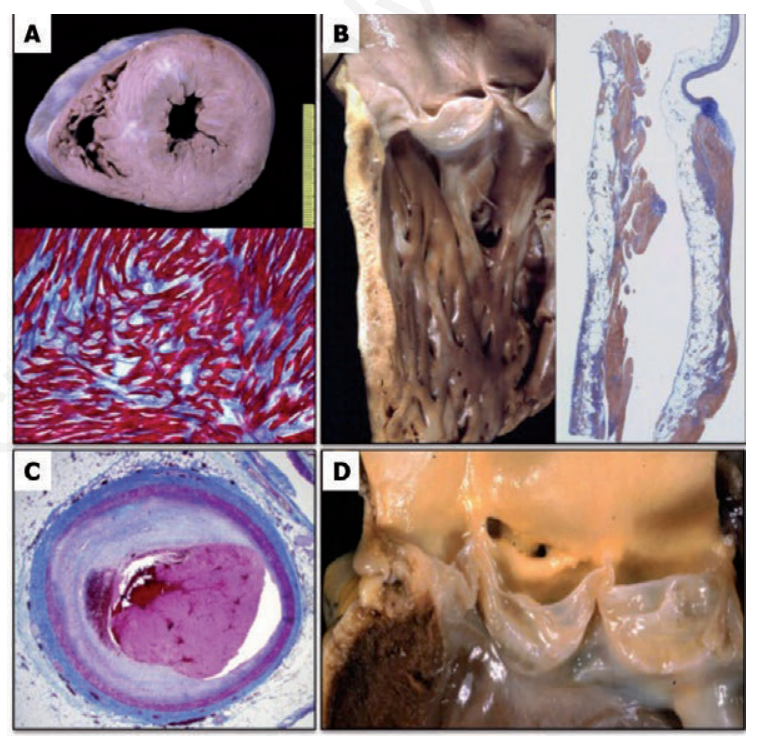

Figure 2. Leading causes of sudden cardiovascular death in young competitive athletes. A) Hypertrophic cardiomyopathy: short-axis cut of the heart specimen showing asymmetric septal hypertrophy with multiple septal scars (top); histology of the interventricular septum revealing typical myocardial disarray with interstitial fibrosis (bottom) (Heidenhain's trichrome); B) arrhythmogenic right ventricular cardiomyopathy: section of the heart specimen along the right ventricular infundibulum (left); panoramic histological view of the right infundibular free wall showing wall thinning with fibro-fatty replacement (right) (Heidenhain's trichrome); C) atherosclerotic coronary artery disease: histology of the proximal tract of the left anterior descending coronary artery showing a non-obstructive fibrous plaque complicated by luminal thrombosis due to endothelial erosion (Heidenhain's trichrome); D) congenital coronary anomaly: gross view of the aortic root showing both coronary ostia located in the right coronary sinus, with left coronary artery arising from the right aortic sinus of Valsalva and running between the aorta and the pulmonary trunk. Adapted from Corrado et al., $2011^{34}$ with permission. 
biopsy; ii) morpho-functional abnormalities consisting of regional RV wall motion abnormalities (regional akinesia, dyskinesia or bulging) plus RV dilatation or global RV dysfunction; iii) depolarization abnormalities such as epsilon waves, delayed S-wave upstroke in V1V3 and late potentials on signal-averaged ECG; iv) T-wave inversion; v) ventricular arrhythmias; and vi) positive family history of ARVC or SD and/or identification of a pathogenetic mutation by molecular genetic testing. ${ }^{44} \mathrm{ARVC}$ has been demonstrated to be a leading cause of SD in young people and athletes of the Veneto region of Italy (up to $20 \%$ of cases), whereas in the USA it is an uncommon substrate. ${ }^{5,8,45}$ This finding may depend on the experience of the pathologist or coroner who performs post-mortem investigation as ARVC is rarely associated with cardiomegaly and may spare the LV, so that the affected heart may be erroneously diagnosed as normal. Of note, in a British study on SD in athletes, whose hearts were referred for post-mortem to a tertiary center, the prevalence of ARVC was similar (13\%) to that found in the Italian series. ${ }^{46}$ The left dominant variant of ARVC is characterized by an early and predominant LV involvement, as a result of a specific genetic background. ${ }^{47}$ At variance with the classic rightdominant variant, the power of traditional investigations including routine ECG and standard echocardiography for the diagnosis of left-dominant ARVC is limited because repolarization abnormalities and LV systolic dysfunction, either regional or global, are observed in a minority of affected patients. The reason is that the fibro-fatty scarring process predominantly involves the sub-epicardial and mid-mural myocardial layers, which contribute less to ventricular contraction, and the disease lesion can only be identified in the form of late gadolinium enhancement using contrast-enhanced cardiac magnetic resonance imaging. ${ }^{48}$ Not surprisingly, the incidence of SD in patients with the classic ARVC variant has markedly decreased since the introduction of ECG preparticipation screening, while the difficultto-diagnose left dominant variant is now an increasingly emergent substrate at post-mortem. ${ }^{49,50}$ Competitive sports increase the risk of SD by 5 -fold in adolescent and young adults with ARVC. ${ }^{5}$ Sports have also been implicated as the most important environmental factor promoting ARVC progression and worsening of the disease arrhythmic substrate. Actually, geneticallydetermined impairment of cell-to-cell adhesion may predispose to myocyte detachment and death, mostly under condition of increased mechanical wall stress such that occurring during competitive sports. ${ }^{51}$ Kirchhof et al. ${ }^{52}$ demonstrated that in heterozygous plakoglobin-deficient mice, endurance training accelerated the development of right ventricular abnormalities and ventricular arrhythmias. James et al..$^{53}$ and Saberniak et al..$^{54}$ confirmed in clinical studies that endurance sports and intense physical exercise increase age-related penetrance, risk of ventricular tachyarrhythmias and occurrence of heart failure in
ARVC desmosomal gene carriers. Ruwald et al. ${ }^{55}$ reported that the absolute risk of malignant arrhythmic events in ARVC patients who participated in competitive sports reached $61 \%$ at 40 years of age. On the other hand, early identification of affected patients by preparticipation screening and disqualification from competitive sports offers the potential to prevent SD. ${ }^{3}$ Accordingly, both European and American recommendations for sports eligibility in patients with heart diseases and the International Task Force consensus document on ARVC treatment agree that restriction from competitive sports of patients with ARVC should be regarded as a therapeutic measure aimed to reduce the risk of SD. ${ }^{56-58}$

\section{Premature coronary artery disease}

Premature coronary atherosclerosis is an important substrate for SD even in young people and athletes (Figure 2C). In these young subjects, coronary artery disease exhibits distinctive characteristics in terms of warning clinical prodromal and pathological features, such as extent, site, and morphology of obstructive atherosclerotic plaques. ${ }^{59,60}$ Young individuals dying from premature coronary artery disease usually do not have history of angina pectoris or previous myocardial infarction and SD is often the first manifestation of the disease. Exercise testing may fail to show myocardial ischemia or arrhythmias. Fatal coronary atherosclerosis most often presents as a single vessel disease that characteristically affects the proximal left anterior descending coronary artery and is often due to fibrocellular plaques (i.e., fibrous plaques with intimal smooth muscle cell hyperplasia, so-called accelerated atherosclerosis) and a preserved tunica media in the absence of acute thrombosis. These morphological features have been suggested to underlie abnormal hypervasoreactivity, possibly culminating in cardiac arrest by vasospastic myocardial ischemia. Because of the scarcity of warning symptoms and the limitation of exercise testing, early identification of young subjects/athletes with premature coronary atherosclerosis at risk of ischemic cardiac arrest remains a challenge.

\section{Congenital coronary artery anomalies}

Anomalous origin of a coronary artery from the wrong coronary sinus is a congenital malformation with a silent clinical course, which may precipitate sudden and unexpected ischemic cardiac arrest in young competitive athletes (Figure 2D). ${ }^{61,62}$ The most frequent anatomic findings consist of both (left and right) coronary arteries arising either from the right or the left coronary sinus. In both conditions, as the anomalous coronary vessel leaves the aorta, it adopts an acute angle with the aortic wall, and, thus, traverses between the aorta and the pulmonary trunk, often following an aortic intramural course, with a slit-like lumen. ${ }^{63,64}$ Exercise-induced myocardial 
ischemia has been hypothesized to be caused by aortic root expansion, which compresses the anomalous vessel against the pulmonary trunk, increases the acute angulation of the coronary takeoff, and aggravates the slit-like shape of the lumen of the proximal intramural portion of the aberrant coronary vessel.

Congenital coronary anomalies are most often undetectable by ECG screening, but they should be suspected in young individuals or athletes who complain of effort-induced either chest pain or syncope. Although echocardiographic examination may evidence the aberrant coronary artery, definitive diagnosis relies on imaging tests such as cardiac MR or CT-coronary angiography, which allow to accurately demonstrate the anomalous coronary artery origin and course.

\section{Mitral valve prolapse}

Mitral valve prolapse has been largely underestimated as a cardiac pathology responsible of $\mathrm{SD}$ in the athlete by general and forensic pathologists. This may be explained by the traditional perspective that MVP is a common valve variant with an estimated prevalence in the general population of 2$3 \%$. Hence, it is deemed to be an uncertain or unlikely cause of SD at post-mortem investigation. Although it is generally regarded as a benign condition, malignant ventricular arrhythmias and SD have been reported; the incidence of SD in MVP has been estimated to range from $0.2 \% / y$ to $0.4 \% / y$ in prospective follow-up studies. ${ }^{65}$ Using rigorous criteria for morphologic diagnosis (i.e., myxomatous mitral valve) at post-mortem, MVP has been implicated in $7 \%$ of all cases of juvenile SD in the Registry of the Veneto region of Italy. Recent clinicopathologic studies on MVP patients with arrhythmias or SD victims provided new insights into our understanding of the underlying myocardial substrate. ${ }^{66}$ Revision of the histologic features in SD victims demonstrated the presence of myocardial fibrosis affecting both the inferobasal LV free wall (under the posterior leaflet) and the papillary muscle (Figure 3). The scarring process may be caused by the mechanical stretch of the inferobasal wall and papillary muscles secondary to the excessive mobility of the valve leaflets caused by posterior systolic curling, eventually leading to myocardial hypertrophy and scarring. ${ }^{67}$ Most important, the presence and location of myocardial fibrosis observed at histology in SD victims was confirmed in living patients with MVP and ventricular arrhythmias by demonstration of late gadolinium enhancement at cardiac magnetic resonance. ${ }^{66,67}$ This suggests a promising role of cardiac magnetic resonance for arrhythmic risk stratification beyond traditional electrophysiological markers. The athlete with at-risk arrhythmic MVP can be identified at preparticipation screening based on the following characteristics: a young adult (most often woman) with a mid-systolic click at auscultation, T-wave abnormalities on inferior leads, complex ventricular arrhythmias with a right bundle branch block morphology on ECG, and bi-leaflet involvement of the mitral valve on echocardiography. These clinical features may help to select a high-risk subgroup of patients, which are candidates for further evaluation by cardiac magnetic resonance.
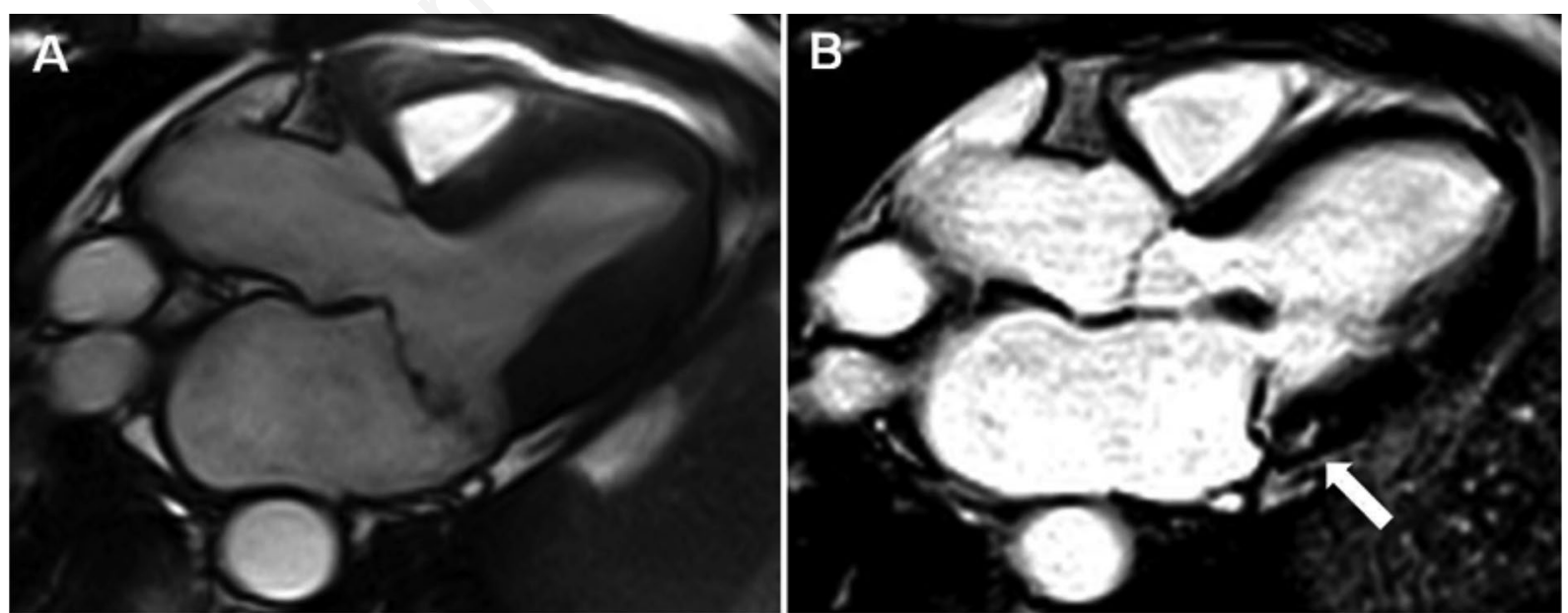

Figure 3. Cardiac magnetic resonance findings in a young woman who survived out-of-hospital cardiac arrest secondary to arrhythmic mitral valve prolapse. Out-of-hospital cardiac arrest occurring in a 29-year-old female with arrhythmic mitral valve prolapse. Cardiac magnetic resonance (three chambers, long axis view) showed thickening and prolapse of both mitral valve leaflets (A) and evidence of mid-myocardial late gadolinium enhancement affecting the basal inferolateral left ventricular wall behind the prolapsing posterior mitral valve leaflet (B, arrow). 


\section{Myocarditis}

Either in its acute or healed forms, myocarditis may provide a myocardial electrical substrate for lifethreatening ventricular arrhythmias and SD. Post-mortem studies showed that fatal arrhythmic events may be the result of a focal acute myocarditis which is clinically silent and difficult to be detected by endomyocardial biopsy.

Recent clinical and pathologic studies have demonstrated that an isolated, non-ischemic LV scar, due to either a healed myocarditis or an inherited cardiomyopathy, may be associated with lifethreatening arrhythmias and SD in the athlete. ${ }^{48-50} \mathrm{~A}$ recent post-mortem study reported a $25 \%$ prevalence of isolated, non-ischemic LV scar among the causes of juvenile SD during exercise. ${ }^{49}$ This substrate, which can be evidenced in vivo as late gadolinium enhancement at cardiac magnetic resonance, has been shown to underlie exercise-induced ventricular arrhythmias (Figure 4) ${ }^{48}$ Furthermore, young athletes showing ventricular arrhythmias with a right bundle branch block pattern (suggesting a LV origin) and isolated, non-ischemic LV late-gadolinium enhancement at cardiac magnetic resonance may experience malignant arrhythmic events including $\mathrm{SD} .{ }^{48}$ The diagnostic power of routine cardiovascular tests for this condition is limited because ECG changes and echocardiographic LV systolic dysfunction, either regional or global, are found in a minority of affected patients. The segmental nature of myocardial fibrosis confined to small myocardial area and involving outer wall layers (without reaching the subendocardium which is the part of the LV that contributes the most to myocardial thickening) explains why the scar is usually undetectable by echocardiography. ${ }^{48}$ The difficulty to identify affected athletes by preparticipation screening makes this condition one of most challenging and unpredictable causes of SD during sports.

\section{Other structural causes}

Ventricular pre-excitation syndrome (WPW syndrome) or progressive cardiac conduction disease (Lenègre's disease) may represent an uncommon substrate for exercise-related SD.

\section{Non-structural causes}

A sizeable proportion of young people and athletes who died suddenly (ranging from 5 to $25 \%$ of the reported series) have no evidence of structural heart diseases and the cause of their cardiac arrest is in all likelihood related to a primary electrical heart disease such as inherited cardiac ion channel defects (channelopathies), including long and short QT syndromes, Brugada syndrome and catecholaminergic polymorphic ventricular tachycardia. ${ }^{3-29}$

Long QT syndrome is a genetic disorder characterized by prolonged ventricular repolarization (prolonged QTc interval) that predisposes to lifethreatening ventricular arrhythmias such as torsade de pointes, which may degenerate into FV/SD. The genetic background resides on defective genes encoding ion channels that regulate myocyte action potential. According to molecular genetics, different variants of long QT syndrome can be considered. ${ }^{68}$ Mutations of genes that encode potassium channels, either IKs (LQT1) or IKr (LQT2), reduce potassium current that, in turn, accounts for prolongation of repolarization phase of the action potential and lengthening of QT interval. LQT3 is caused by mutations of $S C N 5 \mathrm{~A}$ gene (sodium channel gene) that alter the sodium channel inactivation with persistence of a small inward current that causes prolongation of early phase of repolarization. Different molecular mechanisms may explain differences seen in clinical manifestations. LQT1 patients are prone to syncope or cardiac arrest during physical exercise, mostly while swimming. On the contrary, LQT3 patients show a bradycardia-dependent QT prolongation and they experience SD events at rest (while sleeping). LQT2 subjects are more susceptible during emotions and under acoustic stimuli. ${ }^{69}$

Brugada syndrome is an inherited ion channel disease characterized by the peculiar ECG pattern consisting of right precordial coved type ST-segment elevation (both spontaneous or induced by pharmacologic sodium channel blockade) in association with arrhythmia-related syncope/cardiac arrest, inducibility at programmed ventricular stimulation, or a familial history of SD. A cardiac sodium channel gene (SCN5A) mutation has been detected in up to $30 \%$ of Brugada syndrome cases. ${ }^{70}$ Ventricular fibrillation leading to SD usually occurs at rest and in many cases at night (during sleep) as a consequence of an increased vagal stimulation and/or withdrawal of sympathetic activity. Enhanced adrenergic drive, such as that occurring during physical activity, could have an inhibitory effect and theoretically reduce the risk of SD. On the other hand, the adaptation of the cardiac autonomic nervous system to systematic training, which results in increased resting vagal tone or during the postexercise recovery period may enhance the propensity of athletes with Brugada syndrome to die at rest, during sleep, or immediately after effort. ${ }^{71}$

Catecholaminergic ventricular tachycardia is an inherited ion channel disease characterized by exercise-induced polymorphic ventricular tachycardia (most often with the so-called bidirectional pattern) and the propensity to cardiac arrest due to VF. ${ }^{72}$ Unlike long QT syndrome and Brugada syndrome, this condition is not associated with abnormalities of basal ECG and remains unrecognized unless the athlete undergoes ECG stress testing. A genetically defective ryanodine receptor has been reported to account for an abnormal calcium release from the 
sarcoplasmic reticulum. Accordingly, the potential arrhythmogenic mechanism is triggered activity due to late after-depolarizations, which are provoked by intracellular calcium overload and enhanced by adrenergic stimulation such as that occurring during sports exercise. ${ }^{73}$

\section{Prevention of sudden death by cardiovascular screening}

Cardiovascular medical evaluation of young populations offers the ability to identify asymptomatic individuals who have potentially lethal cardiovascular abnormalities, and to protect them from the risk of SD. ${ }^{74-78}$

Italy is the only country in the world where law mandates that every young subject engaged in competitive sports must undergo a clinical evaluation to obtain eligibility before entering in competitive sports. ${ }^{79}$ A nationwide mass preparticipation screening program, essentially based on 12-lead ECG, has been in practice since 1982. ${ }^{3,76,77}$ A flow chart illustrating the Italian screening protocol is reported in Figure 5. ${ }^{76}$ The initial cardiovascular evaluation consists of complete personal and family history, physical examination including blood pressure measurement, and 12-lead ECG.
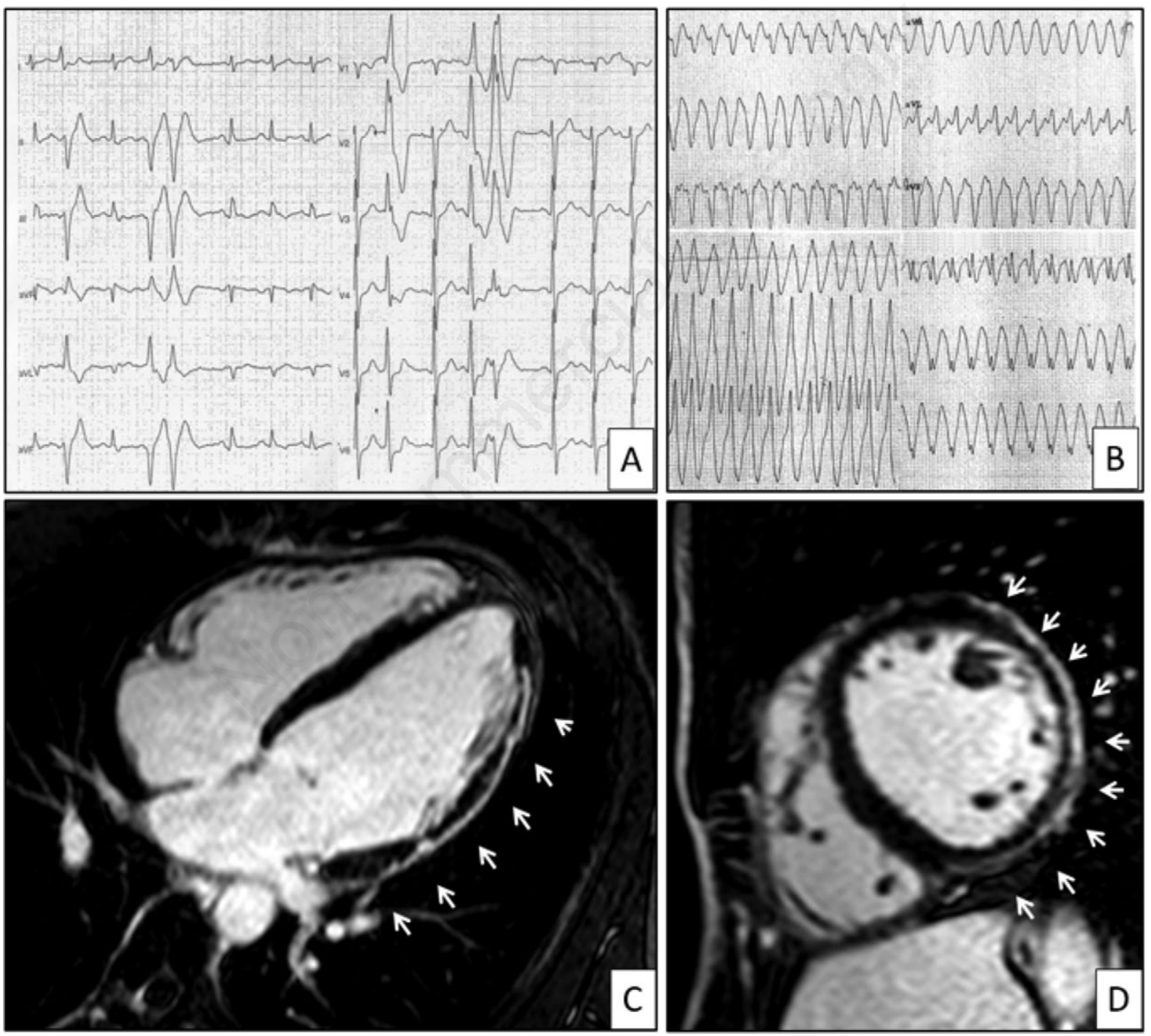

Figure 4. Life-threatening ventricular arrhythmias in an athlete with non-ischemic left ventricular scar. A) A 42 yearsold martial art player presenting with frequent and coupled premature ventricular beats with right bundle branch block/superior axis morphology during exercise testing; B) the athlete experienced sustained ventricular tachycardia during follow-up; $\mathrm{C}$ and D) contrast-enhanced cardiac magnetic resonance revealed a subepicardial/midmyocardial stria of late-gadolinium enhancement involving the antero-lateral, lateral and inferolateral left ventricular wall. Adapted from Zorzi et al., $2016^{48}$ with permission. 
Medical history: The majority of conditions at risk of SD during sports are genetically-determined diseases with an autosomal dominant pattern of inheritance, hence the importance of family history in identifying affected athletes. The family history is considered positive when close relative(s) had experienced a premature heart attack or SD $(<55$ years of age in males and $<65$ years in females), or in the presence of a family history of cardiomyopathy, Marfan syndrome, long QT syndrome, Brugada syndrome, severe arrhythmias, coronary artery disease, or other disabling cardiovascular diseases. The personal history is considered positive in case of exertional chest pain or discomfort, syncope or near-syncope, irregular heartbeat or palpitations, and in the presence of shortness of breath, or fatigue out of proportion to the degree of exertion.

Physical examination: Positive physical findings include musculoskeletal and ocular features suggestive of Marfan syndrome, diminished and delayed femoral artery pulses, mid-or end-systolic clicks, a second heart sound single or widely split and fixed with respiration, marked heart murmurs (any diastolic and systolic grade $\geq 2 / 6$ ), irregular heart rhythm, and brachial blood pressure $>140 / 90 \mathrm{mmHg}$ (on $>1$ readings).

ECG: Twelve-lead ECG is considered positive in the presence of one or more of the findings reported in Table $2 .^{77}$

The screening starts at the beginning of competitive athletic activity that, for the majority of sports disciplines, corresponds to an athlete's age of 12-14 years. Preparticipation evaluation is repeated on a regular basis (every one or two years) in order to timely identify progression over the time of some diseases.

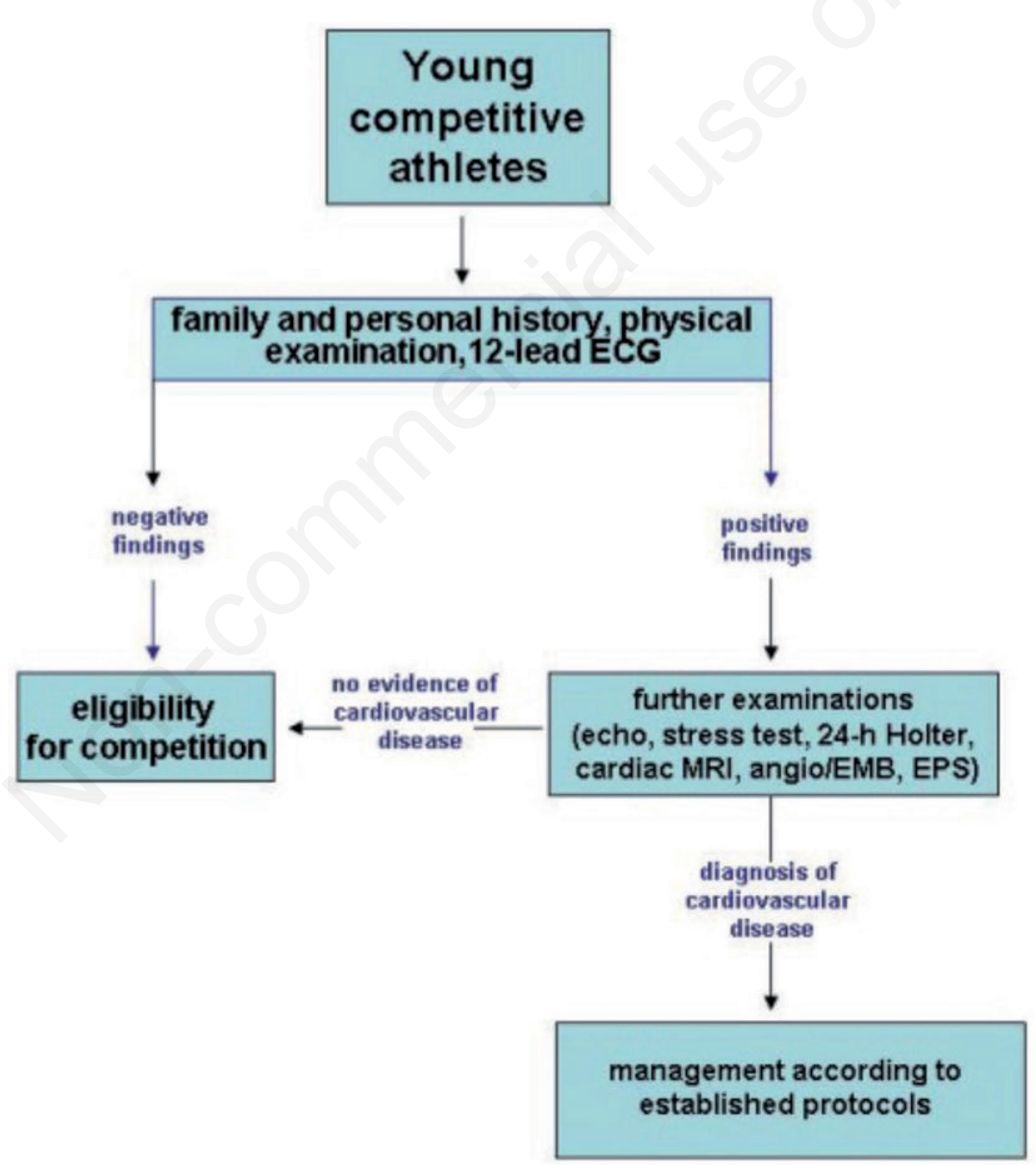

Figure 5. Protocol of the Italian cardiovascular screening of young competitive athletes. The initial cardiovascular evaluation consists of complete personal and family history, physical examination including blood pressure measurement, and 12-lead electrocardiogram (ECG). Subjects who have positive findings at basal evaluation are referred for additional testing, initially non-invasive such as echocardiography, 24-h ambulatory Holter monitoring, and exercise testing. Alternatively, or in uncertain cases invasive tests such as contrast ventriculography (both right and left), coronary angiography, endomyocardial biopsy, and electrophysiologic study may be necessary in order to confirm or rule out suspected heart disease. Finally, subjects recognized to be affected by cardiovascular conditions potentially responsible for sudden death in association with exercise and sport participation are managed according to the available recommendations for sports eligibility. Adapted from Corrado et al., $2008^{76}$ with permission. 


\section{Efficacy of cardiovascular screening}

HCM has been reported to be the leading cause of $\mathrm{SD}$ in young competitive athletes, accounting for up to $40 \%$ of athletic field deaths in the USA. ${ }^{8}$ Although echocardiography is the main diagnostic tool for recognition of $\mathrm{HCM}$, it is expensive and impractical for screening large athletic population. The Italian experience demonstrated that a protocol utilizing ECG in addition to history and physical examination successfully identifies HCM in the general population of young competitive athletes. ${ }^{39}$ Among 33,735 athletes undergoing preparticipation screening at the Center for Sport Medicine of Padova from 1979 to 1999, $22(0.07 \%)$ showed definitive evidence, both clinical and echocardiographic, of HCM. An absolute value of screening sensitivity for HCM in young competitive athletes cannot be derived from this study, because systematic echocardiographic data were not available. However, this $0.07 \%$ prevalence of HCM in the Caucasian athletic population of the Veneto Region of Italy, that was evaluated by history, physical examination and ECG, is similar to that of $0.1 \%$ reported in young Caucasian individuals in the USA, assessed by echocardiography. ${ }^{80}$ This indicates that Italian screening essentially based on 12-lead ECG may be as sensitive as screening by echocardiography in detecting HCM in the young population. Echocardiographic study in addition to the basal protocol does not seem to significantly improve the efficacy of screening in identifying HCM. Pelliccia et al. did not detect any HCM by routine echocardiographic examination in 4450 elite athletes previously cleared by ECG at preparticipation evaluation. ${ }^{81}$

Twelve-lead ECG offers the potential to detect (or to raise clinical suspicion) lethal conditions (other than HCM) manifesting with ECG abnormalities, such as ARVC, dilated cardiomyopathy, long QT syndromes, Lenègre's disease, Brugada syndrome, short QT syndrome, and WPW syndrome. Overall these conditions (including HCM) account for up to two thirds of SD in young athletes.

On the other hand, the possibility of detecting young athletes with either premature coronary atherosclerosis or anomalous coronary artery is limited by the scarcity of baseline ECG signs of myocardial ischemia.

\section{Mortality reduction}

It is noteworthy that none of the young athletes with HCM who were identified in the Padua country area by preparticipation athletic screening and disqualified from training and competition died during an average 8-year follow-up period. ${ }^{39}$ This favorable long-term outcome of former athletes with HCM was the result not only of restriction from competitive sports but also of the subsequent close follow-up and clinical management aimed to prevent sudden death.
These findings suggest that preparticipation screening does not change merely the mode of death, from exercise-related to exercise-unrelated death, but it reduces mortality from HCM.

Subsequently, a time-trend analysis of the changes in incidence rates and causes of SD in young people and athletes age 12 to 35 years in the Veneto region of Italy between 1979 and 2004, definitively demonstrated that preparticipation ECG screening is a life-saving strategy. ${ }^{3}$ According to this time-trend analysis, SD mortality from any cardiovascular cause declined in Italian athletes sharply after the introduction of a nationwide screening program in 1982. During the study periods there were 55 SD in screened athletes (1.9 deaths/100,000 person-years) and 265 deaths in unscreened non-athletes $(0.79$ deaths $/ 100,000$ person-years). The annual incidence of SD in athletes decreased approximately by $90 \%$, from $3.6 / 100,000$ person-years in $1979-1980$ to $0.4 / 100,000$ person-years in 2001-2004, whereas the incidence of SD among the unscreened nonathletic population did not change significantly over that time (Figure 6$).{ }^{3}$ The decline in the death rate started after mandatory screening was launched and persisted to the late screening period. Compared with the pre-screening period (1979-1981), the relative risk of SD was 44\% lower in the early screening period (1982-1992) and $79 \%$ lower in the late screening period (1993-2004). Most of the reduced death rate was due to fewer cases of SD from cardiomyopathies. Over the same time interval, a parallel study examined trends in cardiovascular causes of disqualification from competitive sports in 42,386 athletes undergoing preparticipation screening at the Center for Sports Medicine in Padua. The decline of mortality from cardiomyopathies paralleled the concomitant increase in the number of athletes with cardiomyopathies (both HCM and ARVC) who were identified by preparticipation screening and disqualified from competitive sports.

Most important, during the late-screening-period the annual incidence of SD in screened athletes became equal (in the 1999-to-2000 period) and decreased by $50 \%$ (in the 2001-to-2004 period) compared with that observed in unscreened nonathletes (Figure 6). This finding suggests that the extension of ECG screening to the non-athletic general population of young people offers the possibility to further reduce SD mortality in the young.

These data on the mortality reduction after the implementation of the Italian preparticipation screening program are based on the SD database in the Veneto region of Italy which is unique, because cases are collected according to a prospective study design with systematic investigation of all young people $(\leq 35$ years) who died suddenly and underwent a standardized investigation of the heart by a team of cardiovascular pathologists. The heart specimens, as well as the clinical records of all SD victims since 


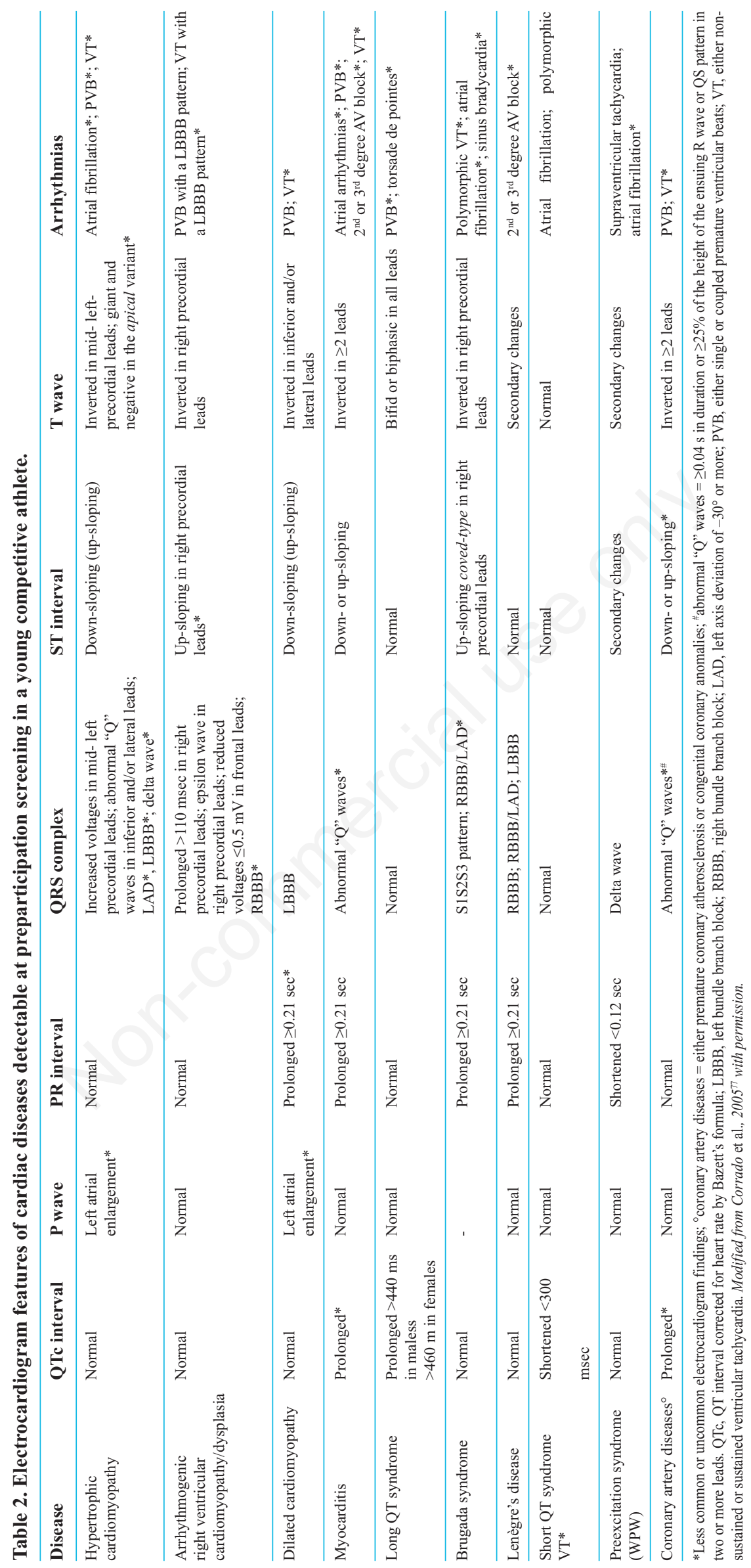


1979, are stored in the Registry of Cardiovascular Pathology, University of Padua, allowing retrieval and review of each case. This accounts for the greater reliability of data on causes and trends of SD in Italian young people and athletes, compared with reports from other countries, which are based on a less rigorous data collection. ${ }^{82-84}$

\section{Cost-benefit considerations}

The young age of the screened athletic population and the genetic nature of the causes of SD in this agegroup profoundly impacts cost-benefit considerations. Unlike older patients with coronary artery diseases or heart failure, adolescents and young adults diagnosed with a genetic disease at risk of arrhythmic SD will survive for many decades with normal or nearly normal life expectancy, thanks to restriction from competition and prophylactic therapy against life-threatening arrhythmias. ${ }^{85}$ This large amount of life-years saved influences costeffectiveness analysis and explains why all reports on ECG screening of young individuals have provided cost estimates per year of life saved well below 50,000 USD, which is the traditional threshold to consider a health intervention as cost-effective. ${ }^{86}$ The benefit of preparticipation evaluation goes beyond the detection of index athletes with an inherited heart disease because it enables cascade screening of relatives and results in a multiplier effect for identifying other affected family members and saving additional lives.

\section{Prevention of sudden death by automatic external defibrillator}

The screening sensitivity for cardiovascular disorder responsible of SD in the young is not $100 \%$. The screening ability to detect young competitive athletes with either premature coronary atherosclerosis or anomalous coronary artery is limited by the scarcity of previous symptoms and baseline ECG signs of myocardial ischemia. ${ }^{34}$ Moreover, there are other atrisk substrates such as early/minor forms of cardiomyopathies and segmental, non-ischemic LV fibrosis that can be missed by routine cardiovascular evaluation. This justifies the growing efforts to implement secondary prevention strategy based on early external defibrillation of unpredictable arrhythmic cardiac arrest. In fact, the most important factor influencing survival from sudden cardiac arrest is the access to rapid defibrillation through on-site automatic external defibrillator (AED). Public access to AEDs has been successful in improving survival (up to $52 \%$ ) from out-of-hospital cardiac arrest in many settings including casino, airlines, and airports. These favorable results were obtained in individuals with a mean age $>60$ years that most likely experienced an ischemic cardiac arrest due to atherosclerotic coronary artery disease. Limited research is available regarding early defibrillation programs for prevention of SD in young people and athletes. Concerns have been raised about the effectiveness of early defibrillation of sudden cardiac arrest occurring in the young athletic

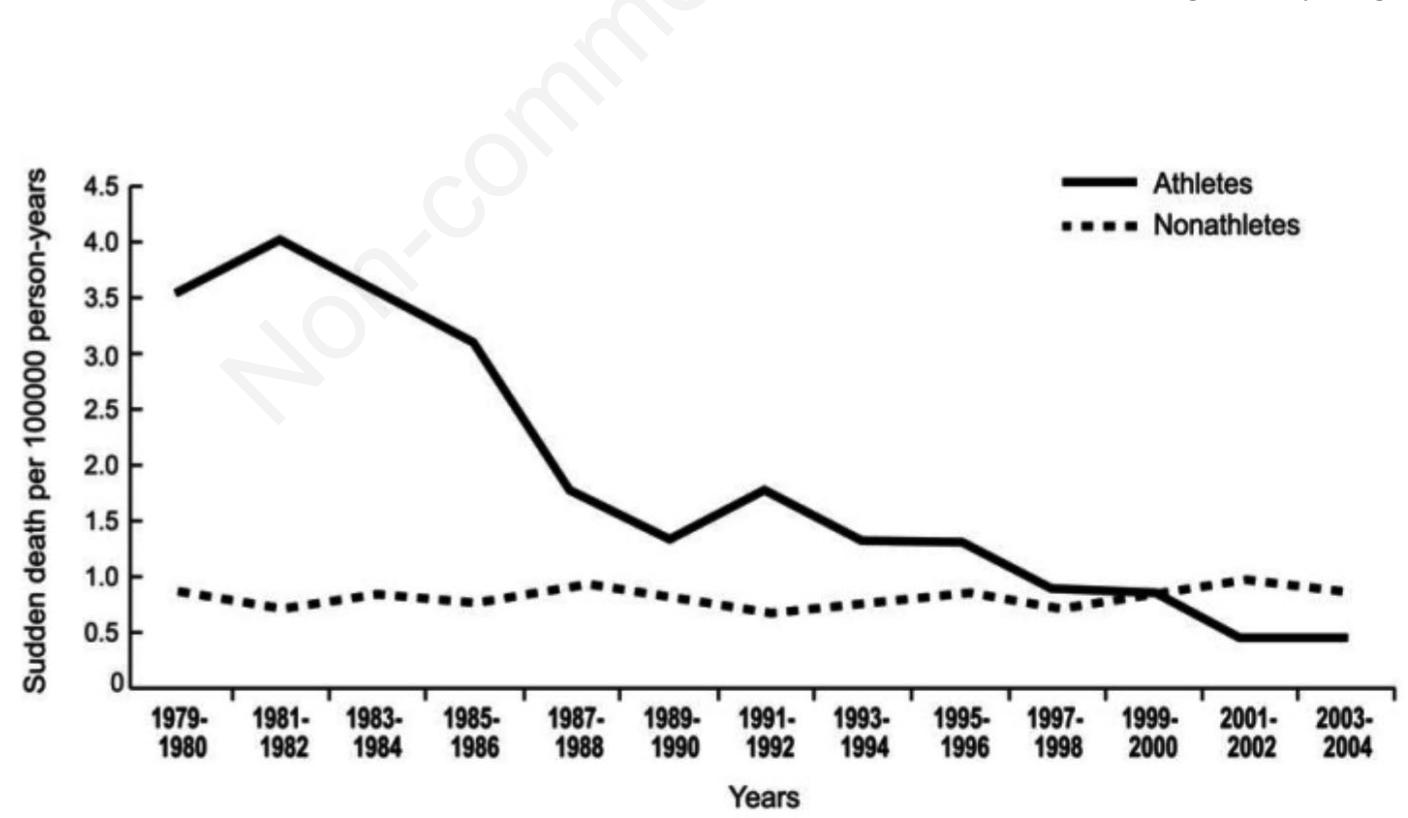

Figure 6. Annual incidence rates of sudden death (SD) among screened competitive athletes and unscreened non-athletes 12-35 years of age in the Veneto Region of Italy. During the study period (the nationwide pre-participation screening program was launched in 1982), the annual incidence of SD in screened athletes declined by $89 \%$ (P for trend $<0.001$ ); in contrast, the incidence rate of SD in unscreened non-athletes did not demonstrate consistent changes over time. Note that during late-screening-period, the annual incidence of sudden cardiovascular death in screened athletes became equal in the 1999-to-2000 period and lower than that observed in unscreened non-athletes in the 2001-to-2004 period). Adapted from Corrado et al., $2006^{3}$ with permission. 
population with different causes of cardiac arrest, mostly consisting of cardiomyopathies, compared with older people suffering VF from coronary artery disease. Drezner et al. reported the experience on 1710 US high schools with free-standing AED program and demonstrated an improved survival rate for young people and athletes with sudden cardiac arrest if early defibrillation is achieved. ${ }^{87}$ Twenty-three of the 36 sudden cardiac arrest victims (64\%) survived to hospital discharge, including 9 of 14 students-athletes (64\%) and 14 of 22 older non-students (64\%). Although this was a retrospective cohort study, the results of successful early defibrillation are very encouraging and support the implementation of AED programs to prevent SD in young people and athletes.

\section{Future perspectives}

The future for prevention of SD in young people and athletes relies on continuing efforts to better understand the substrates and mechanisms underlying arrhythmic cardiac arrest, with particular reference to genetic disorders predisposing to ventricular fibrillation in the absence of structural heart disease.

The extraordinary advances in molecular genetics during the last two decades have led to identification of a growing number of defective genes involved in the pathogenesis of inherited cardiomyopathies, including cardiac ion channel disorders. It is hoped that in the near future genetic molecular tests for definitive preclinical diagnosis of heart muscle diseases at risk of sudden cardiac death in the young will be clinically available, in order to allow more focused and efficient preventive strategies.

\section{References}

1. Corrado D, Zorzi A. Sudden death in athletes. Int J Cardiol 2017;237:67-70.

2. Harmon KG, Zigman M, Drezner JA. The effectiveness of screening history, physical exam, and ECG to detect potentially lethal cardiac disorders in athletes: a systematic review/meta-analysis. J Electrocardiol 2015; 48:329-38.

3. Corrado D, Basso C, Pavei A, et al. Trends in sudden cardiovascular death in young competitive athletes after implementation of a preparticipation screening program. JAMA 2006;296:1593-601.

4. Maron BJ, Gohman TE, Aeppli D. Prevalence of sudden cardiac death during competitive sports activities in Minnesota high school athletes. J Am Coll Cardiol 1998; $32: 1881-4$.

5. Corrado D, Basso C, Rizzoli G, et al. Does sports activity enhance the risk of sudden death in adolescents and young adults? J Am Coll Cardiol 2003;42:1959-63.

6. Gerein RB, Osmond MH, Stiell IG, et al. What are the etiology and epidemiology of out-of-hospital pediatric cardiopulmonary arrest in Ontario, Canada? Acad Emerg Med 2006;13:653-8.

7. Ong ME, Stiell I, Osmond MH, et al. Etiology of pediatric out-of-hospital cardiac arrest by coroner's diagnosis. Resuscitation 2006;68:335-42.
8. Maron BJ, Doerer JJ, Haas TS, et al. Sudden deaths in young competitive athletes: analysis of 1866 deaths in the United States, 1980-2006. Circulation 2009;119:1085-92.

9. Chugh SS, Reinier K, Balaji S, et al. Population-based analysis of sudden death in children: The Oregon Sudden Unexpected Death Study. Heart Rhythm 2009; 6:1618-22.

10. Atkins DL, Everson-Stewart S, Sears GK, et al. Epidemiology and outcomes from out-of-hospital cardiac arrest in children: the Resuscitation Outcomes Consortium Epistry-Cardiac Arrest. Circulation 2009; 119:1484-91.

11. Solberg EE, Gjertsen F, Haugstad E, Kolsrud L. Sudden death in sports among young adults in Norway. Eur J Cardiovasc Prev Rehabil 2010;17:337-41.

12. Holst AG, Winkel BG, Theilade J, et al. Incidence and etiology of sports-related sudden cardiac death in Denmark: implications for preparticipation screening. Heart Rhythm 2010;7:1365-71.

13. Donohoe RT, Innes J, Gadd S, et al. Out-of-hospital cardiac arrest in patients aged 35 years and under: a 4year study of frequency and survival in London. Resuscitation 2010;81:36-41.

14. Park CB, Shin SD, Suh GJ, et al. Pediatric out-ofhospital cardiac arrest in Korea: A nationwide population-based study. Resuscitation 2010;81:512-7.

15. Deasy C, Bernard SA, Cameron P, et al. Epidemiology of paediatric out-of-hospital cardiac arrest in Melbourne, Australia. Resuscitation 2010;81:1095-100.

16. Harmon KG, Asif IM, Klossner D, Drezner JA. Incidence of sudden cardiac death in National Collegiate Athletic Association athletes. Circulation 2011;123:1594-600.

17. Winkel BG, Holst AG, Theilade J, et al. Nationwide study of sudden cardiac death in persons aged 1-35 years. Eur Heart J 2011;32:983-90.

18. Meyer L, Stubbs B, Fahrenbruch C, et al. Incidence, causes, and survival trends from cardiovascular-related sudden cardiac arrest in children and young adults 0 to 35 years of age: a 30-year review. Circulation 2012;126: 1363-72.

19. Margey R, Roy A, Tobin S, et al. Sudden cardiac death in 14- to 35-year olds in Ireland from 2005 to 2007: a retrospective registry. Europace 2011;13:1411-8.

20. Eckart RE, Shry EA, Burke AP, et al. Sudden death in young adults: an autopsy-based series of a population undergoing active surveillance. J Am Coll Cardiol 2011; 58:1254-61.

21. Roberts WO, Stovitz SD. Incidence of sudden cardiac death in Minnesota high school athletes 1993-2012 screened with a standardized pre-participation evaluation. J Am Coll Cardiol 2013;62:1298-301.

22. Pilmer CM, Kirsh JA, Hildebrandt D, et al. Sudden cardiac death in children and adolescents between 1 and 19 years of age. Heart Rhythm 2014;11:239-45.

23. Pilmer CM, Porter B, Kirsh JA, et al. Scope and nature of sudden cardiac death before age 40 in Ontario: a report from the cardiac death advisory committee of the office of the chief coroner. Heart Rhythm 2013;10:517-23.

24. Berdowski J, de Beus MF, Blom M, et al. Exerciserelated out-of-hospital cardiac arrest in the general population: incidence and prognosis. Eur Heart J 2013; 34:3616-23.

25. Maron BJ, Haas TS, Ahluwalia A, Rutten-Ramos SC. Incidence of cardiovascular sudden deaths in Minnesota high school athletes. Heart Rhythm 2013;10:374-7.

26. Risgaard B, Winkel BG, Jabbari R, et al. Burden of sudden cardiac death in persons aged 1 to 49 years: nationwide study in Denmark. Circ Arrhythm Electrophysiol 2014;7:205-11. 
27. Marijon E, Uy-Evanado A, Reinier K, et al. Sudden cardiac arrest during sports activity in middle age. Circulation 2015;131:1384-91.

28. Harmon KG, Asif IM, Maleszewski JJ, et al. Incidence, cause, and comparative frequency of sudden cardiac death in national collegiate athletic association athletes: a decade in review. Circulation 2015;132:10-9.

29. Grani C, Chappex N, Fracasso T, et al. Sports-related sudden cardiac death in Switzerland classified by static and dynamic components of exercise. Eur J Prev Cardiol 2016;23:1228-36.

30. Basso C, Burke M, Fornes P, et al. Guidelines for autopsy investigation of sudden cardiac death. Virchows Arch 2008;452:11-8.

31. Corrado D, Basso C, Thiene G. Essay: Sudden death in young athletes. Lancet 2005;366:S47-8.

32. Corrado D, Drezner J, Basso C, et al. Strategies for the prevention of sudden cardiac death during sports. Eur J Cardiovasc Prev Rehabil 2011;18:197-208.

33. Mittleman MA, Maclure M, Tofler GH, et al. Triggering of acute myocardial infarction by heavy physical exertion. Protection against triggering by regular exertion. Determinants of Myocardial Infarction Onset Study Investigators. N Engl J Med 1993;329:1677-83.

34. Corrado D, Schmied C, Basso C, et al. Risk of sports: do we need a pre-participation screening for competitive and leisure athletes? Eur Heart J 2011;32:934-44.

35. Elliott PM, Anastasakis A, Borger MA, et al. 2014 ESC Guidelines on diagnosis and management of hypertrophic cardiomyopathy: the Task Force for the Diagnosis and Management of Hypertrophic Cardiomyopathy of the European Society of Cardiology (ESC). Eur Heart J 2014;35:2733-79.

36. Gersh BJ, Maron BJ, Bonow RO, et al. 2011 $\mathrm{ACCF} / \mathrm{AHA}$ guideline for the diagnosis and treatment of hypertrophic cardiomyopathy: a report of the American College of Cardiology Foundation/American Heart Association Task Force on Practice Guidelines. Circulation 2011;124:e783-831.

37. Marian AJ, Braunwald E. Hypertrophic cardiomyopathy: genetics, pathogenesis, clinical manifestations, diagnosis, and therapy. Circ Res. 2017;121:749-70.

38. Zorzi A, Calore C, Vio R, et al. Accuracy of the ECG for differential diagnosis between hypertrophic cardiomyopathy and athlete's heart: comparison between the European Society of Cardiology (2010) and International (2017) criteria. Br J Sports Med 2017 [Epub ahead of print].

39. Corrado D, Basso C, Schiavon M, Thiene G. Screening for hypertrophic cardiomyopathy in young athletes. $\mathrm{N}$ Engl J Med 1998;339:363-9.

40. Corrado D, Link MS, Calkins H. Arrhythmogenic right ventricular cardiomyopathy. N Engl J Med 2017;376: 1489-90.

41. Zorzi A, Rigato I, Bauce B, et al. Arrhythmogenic right ventricular cardiomyopathy: risk stratification and indications for defibrillator therapy. Curr Cardiol Rep 2016;18:57.

42. Corrado D, Basso C, Thiene G, et al. Spectrum of clinicopathologic manifestations of arrhythmogenic right ventricular cardiomyopathy/dysplasia: a multicenter study. J Am Coll Cardiol 1997;30:1512-20.

43. Basso C, Corrado D, Marcus F, et al. Arrhythmogenic right ventricular cardiomyopathy. Lancet 2009;373:1289300 .

44. Marcus FI, McKenna WJ, Sherrill D, et al. Diagnosis of arrhythmogenic right ventricular cardiomyopathy/ dysplasia: proposed modification of the task force criteria. Circulation 2010;121:1533-41.
45. Van Camp SP, Bloor CM, Mueller FO, et al. Nontraumatic sports death in high school and college athletes. Med Sci Sports Exerc 1995;27:641-7.

46. Finocchiaro G, Papadakis M, Robertus JL, et al. Etiology of sudden death in sports: insights from a United Kingdom Regional Registry. J Am Coll Cardiol 2016;67:2108-15.

47. Sen-Chowdhry S, Syrris P, Prasad SK, et al. Leftdominant arrhythmogenic cardiomyopathy: an under-recognized clinical entity. J Am Coll Cardiol 2008;52:2175-87.

48. Zorzi A, Perazzolo Marra M, Rigato I, et al. Nonischemic left ventricular scar as a substrate of lifethreatening ventricular arrhythmias and sudden cardiac death in competitive athletes. Circ Arrhythm Electrophysiol 2016;9.

49. d'Amati G, De Caterina R, Basso C. Sudden cardiac death in an Italian competitive athlete: Pre-participation screening and cardiovascular emergency care are both essential. Int J Cardiol 2016;206:84-6.

50. Basso C, Rizzo S, Pilichou K, et al. Why arrhythmogenic cardiomyopathy is still a major cause of sudden death in competitive athletes despite preparticipation screening? Circulation 2014;130:A20642.

51. Thiene G, Nava A, Corrado D, et al. Right ventricular cardiomyopathy and sudden death in young people. $\mathrm{N}$ Engl J Med 1988;318:129-33.

52. Kirchhof P, Fabritz L, Zwiener M, et al. Age- and training-dependent development of arrhythmogenic right ventricular cardiomyopathy in heterozygous plakoglobindeficient mice. Circulation 2006;114:1799-806.

53. James CA, Bhonsale A, Tichnell C, et al. Exercise increases age-related penetrance and arrhythmic risk in arrhythmogenic right ventricular dysplasia/ cardiomyopathy-associated desmosomal mutation carriers. J Am Coll Cardiol 2013;62:1290-7.

54. Saberniak J, Hasselberg NE, Borgquist R, et al. Vigorous physical activity impairs myocardial function in patients with arrhythmogenic right ventricular cardiomyopathy and in mutation positive family members. Eur J Heart Fail 2014;16:1337-44.

55. Ruwald AC, Marcus F, Estes NA 3rd, et al. Association of competitive and recreational sport participation with cardiac events in patients with arrhythmogenic right ventricular cardiomyopathy: results from the North American multidisciplinary study of arrhythmogenic right ventricular cardiomyopathy. Eur Heart J 2015;36: 1735-43.

56. Corrado D, Wichter T, Link MS, et al. Treatment of arrhythmogenic right ventricular cardiomyopathy/ dysplasia: an International Task Force Consensus Statement. Circulation. 2015;132:441-53.

57. Pelliccia A, Fagard R, Bjornstad HH, et al. Recommendations for competitive sports participation in athletes with cardiovascular disease: a consensus document from the Study Group of Sports Cardiology of the Working Group of Cardiac Rehabilitation and Exercise Physiology and the Working Group of Myocardial and Pericardial Diseases of the European Society of Cardiology. Eur Heart J 2005;26:1422-45.

58. Maron BJ, Udelson JE, Bonow RO, et al. Eligibility and disqualification recommendations for competitive athletes with cardiovascular abnormalities: task force 3 : hypertrophic cardiomyopathy, arrhythmogenic right ventricular cardiomyopathy and other cardiomyopathies, and myocarditis: a scientific statement from the American Heart Association and American College of Cardiology. J Am Coll Cardiol 2015;66:2362-71.

59. Corrado D, Basso C, Poletti A, et al. Sudden death in 
the young. Is acute coronary thrombosis the major precipitating factor? Circulation 1994;90:2315-23.

60. Green AC, Sheppard MN. Sudden cardiac death associated with premature atheroma in the young: an autopsy study emphasising single-vessel lesions. Cardiol Young 2016;26:743-8.

61. Hill SF, Sheppard MN. A silent cause of sudden cardiac death especially in sport: congenital coronary artery anomalies. Br J Sports Med 2014;48:1151-6.

62. Frommelt PC. Congenital coronary artery abnormalities predisposing to sudden cardiac death. Pacing Clin Electrophysiol 2009;32:S63-6.

63. Basso C, Corrado D, Thiene G. Congenital coronary artery anomalies as an important cause of sudden death in the young. Cardiol Rev 2001;9:312-7.

64. Basso C, Maron BJ, Corrado D, Thiene G. Clinical profile of congenital coronary artery anomalies with origin from the wrong aortic sinus leading to sudden death in young competitive athletes. J Am Coll Cardiol 2000;35:1493-501.

65. Nishimura RA, McGoon MD, Shub C, et al. Echocardiographically documented mitral-valve prolapse. Long-term follow-up of 237 patients, N Engl J Med 1985;313:1305-9.

66. Basso C, Perazzolo Marra M, Rizzo S, et al. Arrhythmic mitral valve prolapse and sudden cardiac death, Circulation 2015;132:556-66.

67. Perazzolo Marra M, Basso C, De Lazzari M, et al. Morphofunctional abnormalities of mitral annulus and arrhythmic mitral valve prolapse, Circ. Cardiovasc. Imaging 2016;9:e005030.

68. Nakano Y, Shimizu W. Genetics of long-QT syndrome. J Hum Genet. 2016;61:51-5.

69. Schwartz PJ, Crotti L, Insolia R. Long-QT syndrome: from genetics to management. Circ Arrhythm Electrophysiol. 2012;5:868-77.

70. Antzelevitch C, Brugada P, Borggrefe M, et al. Brugada syndrome: report of the second consensus conference: endorsed by the Heart Rhythm Society and the European Heart Rhythm Association. Circulation 2005;111:659-70.

71. Mascia G, Arbelo E, Hernandez-Ojeda J, Solimene F, et al. Brugada syndrome and exercise practice: current knowledge, shortcomings and open questions. Int J Sports Med 2017;38:e2.

72. Cerrone M, Napolitano C, Priori SG. Catecholaminergic polymorphic ventricular tachycardia: A paradigm to understand mechanisms of arrhythmias associated to impaired $\mathrm{Ca}(2+)$ regulation. Heart Rhythm 2009;6:1652-9.

73. Arias MA, Fernandez-Guerrero JC, Herrador J, Pagola C. Catecholaminergic polymorphic ventricular tachycardia as a cause of sudden death in athletes. Am J Emerg Med 2006;24:253-4.

74. Corrado D, Basso C, Thiene G. Sudden cardiac death in athletes: what is the role of screening? Curr Opin Cardiol 2012;27:41-8.

75. Pelliccia A, Corrado D. Can electrocardiographic screening prevent sudden death in athletes? Yes. BMJ 2010;341:c4923.
76. Corrado D, Basso C, Schiavon M, et al. Preparticipation screening of young competitive athletes for prevention of sudden cardiac death. J Am Coll Cardiol 2008;52:1981-9.

77. Corrado D, Pelliccia A, Bjornstad HH, et al. Cardiovascular pre-participation screening of young competitive athletes for prevention of sudden death: proposal for a common European protocol. Consensus Statement of the Study Group of Sport Cardiology of the Working Group of Cardiac Rehabilitation and Exercise Physiology and the Working Group of Myocardial and Pericardial Diseases of the European Society of Cardiology. Eur Heart J 2005;26:516-24.

78. Sharma S, Estes 3rd NAM, Vetter VL, Corrado D. Clinical decisions: Cardiac screening before participation in sports. N Engl J Med 2013;369:2049-53.

79. Decree of the Italian Ministry of Health, February 18, 1982. Norme per la tutela sanitaria dell'attività sportiva agonistica (rules concerning the medical protection of athletic activity). Gazzetta Ufficiale March 5, 1982:63.

80. Maron BJ, Gardin JM, Flack JM, et al. Prevalence of hypertrophic cardiomyopathy in a general population of young adults. Echocardiographic analysis of 4111 subjects in the CARDIA Study. Coronary Artery Risk Development in (Young) Adults. Circulation 1995; 92:785-9.

81. Pelliccia A, Di Paolo FM, Corrado D, et al. Evidence for efficacy of the Italian national pre-participation screening programme for identification of hypertrophic cardiomyopathy in competitive athletes. Eur Heart J 2006;27:2196-200.

82. Corrado D, Basso C, Schiavon M, Thiene G. Corrado and colleagues reply to Van Brabandt and colleagues. BMJ 2016;354:i3631.

83. Maron BJ, Haas TS, Doerer JJ, et al. Comparison of U.S. and Italian experiences with sudden cardiac deaths in young competitive athletes and implications for preparticipation screening strategies. Am J Cardiol 2009;104:276-80.

84. Steinvil A, Chundadze T, Zeltser D, et al. Mandatory electrocardiographic screening of athletes to reduce their risk for sudden death proven fact or wishful thinking? J Am Coll Cardiol 2011;57:1291-6.

85. Myerburg RJ, Vetter VL. Electrocardiograms should be included in preparticipation screening of athletes. Circulation 2007;116:2616-26.

86. Wheeler MT, Heidenreich PA, Froelicher VF, et al. Costeffectiveness of preparticipation screening for prevention of sudden cardiac death in young athletes. Ann Intern Med 2010;152:276-86.

87. Drezner JA, Rao AL, Heistand J, et al. Effectiveness of emergency response planning for sudden cardiac arrest in United States high schools with automated external defibrillators. Circulation 2009;120:518-25. 